\title{
3-Nitrotyrosine and Soluble Vascular and Intracellular Adhesion Molecule Responses to High-Intensity Interval and Steady-State Moderate-Intensity Exercise
}

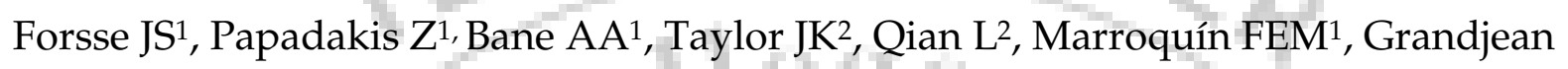
PW 1

${ }^{1}$ Department of Health, Human Performance, and Recreation; Baylor University; Waco, TX

${ }^{2}$ Clinical Laboratory Science; Auburn University-Montgomery; Montgomery, AL

Category: Doctoral

Advisor /Mentor: Grandjean, Peter(Peter_Grandjean@baylor.edu)

ABSTRACT

Vascular endothelium may respond differently to high-intensity interval exercise (HIIE) when compared to moderate-intensity steady state exercise (SSE). We hypothesized that greater sympathetic stimulation of soluble vascular adhesion molecule-1 (sVCAM-1) and intracellular adhesion molecule-1 (sICAM-1) and greater oxidative and nitrative stress on the vascular endothelium may transiently result from HIIE. PURPOSE: Determine the influence of HIIE on sVCAM-1, sICAM-1 and 3-nitrotyrosine (NT), a marker of nitric oxide-dependent reactive nitrogen species and nitrative stress, relative to a comparable amount of moderate-intensity SSE and a dose that is half that of SSE. METHODS: Seventeen male participants (age $27.8 \pm 6.4 \mathrm{yr} ;$ weight $80.6 \pm 9.0 \mathrm{~kg} ;$ BMI $25.1 \pm 2.4 \mathrm{~kg} / \mathrm{m} 2 ; \%$ fat = $19 \pm 5 ; \mathrm{VO} 2 \mathrm{max} 52.1 \pm 7.5 \mathrm{ml} / \mathrm{kg} / \mathrm{min})$ underwent HIIE by treadmill running (90\% and $40 \%$ of VO2reserve in 3:2 min ratio) to expend $500 \mathrm{kcals}$ (H500); HIIE to expend $250 \mathrm{kcals}$ (H250), and; SSE at 70\% VO2reserve to expend $500 \mathrm{kcals}$ (M500) in a randomized crossover design. Intensities of all exercise conditions averaged $70 \%$ VO2reserve. Blood measures of sVCAM-1 (ng/mL), sICAM-1 (ng/mL), NT (nM), epinephrine (EPI) and norepinephrine (NE) in $\mathrm{pg} / \mathrm{mL}$, were obtained just before, immediately after, $2 \mathrm{hr}$ and $24 \mathrm{hr}$ after exercise. Significant differences were determined using 3 by 4 repeated measures ANOVAs. Effect sizes were calculated to determine the magnitude of dependent variable responses to exercise. RESULTS: HIIE resulted in 2 to 2.5 fold greater EPI responses immediately after exercise versus SSE $(p=0.0059$, H250 ES $=1.89$; H500 ES = 3.04). NE increased an average of 5.4 times above pre-exercise values across all exercise conditions ( $p<$ 0.0001). NT decreased immediately after HIIE (H250 ES $=-0.39$; H500 ES $=-0.97$ ) and returned to baseline by $2 \mathrm{hr}$ post-exercise; whereas, NT was elevated $111 \% 2 \mathrm{hr}(\mathrm{ES}=2.46)$ and remained $24 \mathrm{hr}$ after SSE $(\mathrm{p}=$ 0.0001). sVCAM-1 was unchanged with HIIE but increased $6 \%$ immediately following moderate-intensity SSE and remained elevated $24 \mathrm{hr}$ post-exercise $(\mathrm{p}<0.0005, \mathrm{ES}=1.01)$. SUMMARY: Our results are in direct opposition to our hypothesis. Transient elevations in NT and sVCAM-1 after moderate-intensity SSE but not HIIE of similar average intensity and duration may indicate unique effects of interval exercise. NT and sVCAM-1 were not elevated after HIIE in spite of a greater sympathetic response than what was observed after moderate-intensity SSE. 